\title{
THE FUTURE PATHWAYS OF PLACE BRANDING - BRAZILIAN LANDSCAPE
}

iD Fabiana Mariutti Guest Editor - Brazil

Magdalena Florek Guest Editor - Poland

\section{How to cite the article}

American Psychological Association (APA)

Mariutti, F., \& Florek, M. (Eds.). (2022, Jan./Mar.). The future pathways of place branding - Brazilian landscape. Brazilian Journal of Marketing, 21(Special Issues), Editorial, 1-8. https://doi.org/10.5585/remark.v21i1.21486.

"Which Place?" All places, far and near, large, and small, highly developed or on a development path, are looking for the best solutions and practices to fulfil the needs and ambitions of their internal and external stakeholders. Place branding is an approach that supports places in these efforts, strategically and interactively. As Kavaratzis and Florek (2021) claim, "in the near future, the global place branding scholarly community will need to address a series of challenges and questions that remain unsolved and unanswered, with implications for both the theory and the practice" (p.28). At the same time, scholars and practitioners need to consider the regional and local dissimilarities. The Brazilian perspectives, possibilities, and conditions are no exception.

This first edition advances the understanding of this boundless, imperative, and interdisciplinary field of knowledge. We are grateful to the Brazilian Journal of Marketing editorial team, all the authors and reviewers who have contributed to this Special Issue on place branding (Appendix I. Thank-You Note).

Contextually, according to an international literature analysis of the geographical locations stated in the articles on place branding, covering 2014-2018, Europe is the dominant area in the studies, followed by Asia and North America that have also attracted significant 
attention from scholars (Lu et al. 2020). In South America, specifically in Brazil, academic publications on marca de lugar (place brand), gestão de marketing de marca de lugar (place marketing) and gestão de marca de lugar (place branding) are still scarce. Hence there is an urgent need to properly understand the place branding phenomena and disseminate knowledge of it which is often overtaken by practice, causing all sorts of problems and disappointments. Without a solid scientific base, place branding will become and remain merely a response-based tactic.

Therefore, in this Special Issue, we would like to highlight the potential of scientific achievements of academics in Brazil, and to advance the discussion among Brazilian researchers and practitioners. As revealed by Artêncio, Mariutti and Giraldi (2020), a thematic panorama of academic-scientific nature concerns works presented at the main Brazilian Administration conferences: SemeAD, EnANPAD (Marketing Division), and EMA for the last twenty years (1999-2019). With a total of 5,706 studies, only 18 articles address some issues related to place branding or marketing, with the focus mainly on the country level (14), and a few on cities (2) and regions (2). Based on the review of studies' titles, the abstracts, and the keywords identified in the conferences' annals, only three works were identified as revolving around a conceptual designation of place marketing, on presented in SemeAD 2016 and at one EMA 2018; and one of place branding presented at EMA 2018. Notably, the studies are specific to some aspect of place brand (place of origin, identity, image, reputation, and culture to name a few).

In view of this scientific portrait, several conclusions can be drawn.

Firstly, in Brazil research at the national level, place branding studies are certainly evolving. And it needs such a national insertion since the place brand can be used as an instrument in strategic spatial planning to support a structural change in places by contributing to the improvement of the economic and social structure of places and reshaping responses to contemporary challenges faced by the places. Regarding the brand positioning or brand management of a place, a space or territory is not an absolute concept, it is always related to a sphere of social-human application or economic-political structure, which is potentially developed through processes - possibly contested - of producing, maintaining, and assigning meaningful spaces, reinforces Warnaby (2018).

Secondly, there is a need of theoretical and empirical development of the concepts of place brand, place marketing and place branding, not only based on studies on Brazilian cities, states (counties), and regions, but also on other places in the country like airports, avenues, 
beaches, city squares, industrial and other clusters, museums, parks, theatres, etc. Since diverse locus is recognized in a large country like Brazil, providing research questions about the spatiality in marketing literature is key as emphasized by Giovanardi and Lucarelli (2018), Oliveira (2015), and Warnaby (2018).

Thirdly ontological and epistemological advancement of research areas within place branding is necessary to provoke reflections and directions for theoretical advancement, particularly in Brazilian research groups. As Govers (2021) claims, "The funding agencies and governments that appreciate that place branding is of strategic, long-term, and crucial importance are, unfortunately, in a tiny minority" (p.338). With this in mind, we hope to draw attention of Brazilian universities, researchers, and policy makers to the importance and relevance of place branding. Thus, "Place branding has been used to foster economic restructuring, social inclusion and cohesion, political engagement and participation, place identification, and the general well-being of citizens", states Oliveira (2015, p.18).

Stakeholders per se, who are co-creators in the place branding process (Kalandides, Kavaratzis, \& Boisen, 2012; Mariutti \& Giraldi, 2021) should be involved in the management of public and/or private places, strategically aligned with place branding activities to enhance the value of a place (Mariutti \& Giraldi, 2021). Tracing the scope of research along this path establish connections between practice and theory and offers insight into the future directions for specific places, hidden or forgotten in Brazil.

To address the above contexts together with the Brazilian academic setting, we report that 27 papers were submitted during the six-pandemic months (February to August 2021); they were carefully reviewed and analysed. Also, the best studies selected by the track leaders from the Place Branding Track at the Marketing Meeting - EMA 2021 (by Fabiana Mariutti and Mihalis Kavaratzis) and at the EnANPAD Conference 2021 (by Fabiana Mariutti and João Freire) were invited for submission in the regular evaluation process by Brazilian Journal of Marketing. Both conferences are organized by ANPAD - the National Association of Graduate Studies and Research in Administration). For the first time, a place branding track took place at Brazilian conferences. Hence, based on the reviewers' evaluations and research domain comprehension, decisions were taken by the journal's guest editors and editors-in-chief. Finally, nine manuscripts were chosen for publication.

Robust contributions emerged from this pioneer collection. The following systematically chosen papers illustrate some frameworks and insights for place branding research to be conceivably enhanced in national literature. 
The Issue starts with a paper by Carniello and dos Santos who analyse branding as a territorial development strategy, discussing the convergence between city branding and a municipality master plan in the case of São Paulo city. Convergence has been identified between the pillars of the brand and the contents of the master plan, especially the communication as a key element for the social and management propositions. A strategic perspective of place branding is therefore postulated using a qualitative approach, with documental design.

The subsequent paper, by Kamlot and Santos Vieira de Jesus, discusses the core of branding process, mainly, city brand identity. The article contributes to the exploration of the physical and symbolic dimensions of Rio de Janeiro's brand identity and to detailed interpretation thereof, considering the city's recent evolution. Adopting qualitative method to document analysis, the dataset was combined by research papers, e-mails, newsletters, companies and government reports, newspaper articles etc. This study provides important advice on how to design public policies based on the understanding of a city's identity.

The next paper analyses the feelings accompanying content generated by users on Instagram, expressed in hashtags linked to the Pernambuco brand (capital of Recife State). Results of this mixed methods (qualitative and quantitative) research into Lima, Pessanha, Araújo, Alves, and Cesário suggest that studies using sentiment analysis of user content in social networks can be used by public managers to understand residents, tourists, and potential visitors' behaviour through action research, preparing and better adapting the city.

Beck and Ferasso in turn, analyse the reflection of brand identity, namely the image of cities. In this study, a systematic literature review was used to analyse the content of literature about urban image in the context of Southern Common Market (MERCOSUL). The paper demonstrates that the urban image management can lead to how cities can overcome socioeconomic disparities, well-deploying an efficient project of urban image in order to foster a prosperous urban development.

Monte, Pompeu, and Holanda describe an interesting thematic-exploratory qualitative approach to a forgotten property - corporate museum - as a business and cultural space which influences the image of a place. The authors trust that the corporate museums an important cultural asset in promoting the place brand, yet they claim that these museums belong not only to the company but also to the city, region, and country where it is located, attracting tourists and investors, and fostering a sense of belonging among the residents. The study, which was presented at the Place Branding Track at EMA 2021, demonstrates an explanatory and 
theoretical model of how a place brand (city, region, or country) can be boosted by means of a corporate museum.

Another business standpoint was taken by Munaier, Rocha, and Portes as they investigated the impact of the business headquarters location of a physical education service provider on the clients' brand trust. Social exchange theory and brand trust were theoretical foundations for the hypotheses of this quantitative study using multivariate statistical techniques. By realizing that the headquarters location influences the clients' brand trust, the authors suggest that the choice of a location is a complex process that companies should manage carefully; at the same time, places can use these relations for branding purposes.

The measurement issues of the place branding process are discussed by Cunha, Luce, and Rovedder; they have developed and tested the stakeholder-based place brand equity (SBPBE) measure of the Vale dos Vinhedos, a wine region in Southern Brazil. Based on this quantitative research results from the respondents' profiles, the authors demonstrate that the more people's displacements and activities in a place, the more positive their evaluation of the place brand equity tends to be. The outcomes of this research, which was presented at the Place Branding Track at EnANPAD 2021, support a measurement proposition to evaluate these multiple stakeholders on place brand equity based on behavioural data.

The last two papers discuss the research recommendations and directions for future developments in the place branding research domain.

Based on a systematic literature review using Methodi Ordinatio, Campos, Galina, and Giraldi establish the guidelines for cross-disciplinary future research into soft power, analysing its intertwinement with creative industries and place branding. After adopting the content analysis with oblique approach, it was identified six relevant areas with short description and oblique references in their substantial research: psycho-sociology, labour mobility, urban planning, arts diplomacy, gastro-diplomacy, and geographical indications. It is demonstrated how soft power and place branding reveals an explicit relationship with each other theoretically, strategically, and politically.

Ocke and Platt put forward an inviting research agenda providing some advice for scholars, branding practitioners, and marketers taking place from the brand management scope - identity, image, and reputation of a place. By adopting bibliographic material, under a nation branding overview with its economic, political, and cultural-critical orientations, significant proposals for Brazil are suggested to effectively manage place brands, including: (i) appointment of a formal body to coordinate the actions and actors recognizing the role of 
stakeholders in the decision-making stages; (ii) cooperation between governors and leaders to achieve common goals under a unique umbrella branding program that benefit different sectors of the economy; (iii) the conquer of market opportunities by competitive identity assets such as the green place brand orientation; and (iv) application of integrated methods to measure and evaluate the results of place branding efforts.

With this Special Issue, entitled "Which place? The Future Pathways of Place Branding", we hope to encourage actions and stimulate a debate on how place branding can effectively make Brazilian places improved and how the knowledge offered by the academic community can prepare any type of places for the forthcoming challenges. "What comes next?" In consideration of the future role of place branding in Brazil, our recommendations for future studies highlight themes regarding the current status quo of the country, the post-pandemic times, the human re-integration into places, and, indeed, sustainability issues. Research into place branding requires additional integration of disciplines, international partnerships research groups, professional interchanges, public-private initiatives, clusters mapping, geomarketing analysis, and more sophisticated methods of critical appraisal along with well-established instigating research problems.

\section{References}

Artêncio, M., Mariutti, F., \& Giraldi, J. (2020). 20 Anos de Publicações sobre Marca-deLugar no Brasil: um panorama analítico-reflexivo da produção acadêmica em congressos de Administração. In: XXIII Seminários em Administração - SemeAD 2020 - Universidade de São Paulo.

Giovanardi, M., \& Lucarelli, A. (2018). Sailing through marketing: A critical assessment of spatiality in marketing literature. Journal of Business Research, 82: 149-159.

Govers, R. (2021). Chapter 19 - Place branding practice and scholarship: where we are and whither, we may be going. In: Papadopoulos, N., Cleveland, M. "Image, Marketing, and Branding of Places and Place-based Brands: The State of the Art", Edward Elgar.

Kalandides, A., Kavaratzis, M., \& Boisen, M. (2012). From 'necessary evil' to necessity: stakeholders' involvement in place branding. Journal of Place Management and Development, 5 (1): 7-19.

Kavaratzis, M., Florek, M. (2021). Chapter 2 - The who, what, where, when, and why of place branding, In: Papadopoulos, N., Cleveland, M. "Image, Marketing, and Branding of Places and Place-based Brands: The State of the Art", Edward Elgar.

Lu, H., Ma, W., Yang, Q., \& Zhao, P. (2020). Exploring the impact of factors influencing 
case selection in the place branding literature from 2014 to 2018, Journal of Urban Affairs, first online.

Mariutti, F. G., \& Giraldi, J. D. M. E. (2021). Branding cities, regions, and countries: the roadmap of place brand equity. RAUSP Management Journal, 56 (2): 202-216.

Oliveira, E. (2015). Place branding as a strategic spatial planning instrument. Place Branding and Public Diplomacy, 11 (1): 18-33.

Warnaby, G. (2018). Taking a territorological perspective on place branding? Cities, 80: 6466.

\section{Appendix I}

\section{Thank-You Note}

We appreciated the endless support by the Editor in Chief Prof. Dr. Julio Carneiro da Cunha.

We are grateful to following Reviewers, who neatly contributed to our special call:

Dr. Antônio Azevedo - Universidade do Minho, Portugal

Dra. Beatriz Casais - Universidade do Minho, Portugal

Dra. Caroline Finocchio - Universidade Federal do Mato Grosso do Sul, Brazil

Dra. Cecilia Pasquinelli - Università degli Studi di Napoli Parthenope, Italy

Ph.D. Candidate: Donizete Ferreira Beck - Universidade Nove de Julho, SP, Brazil

Dr. Eduardo de Paula e Silva Chaves - Universidade de São Paulo \& Instituto Federal de São

Paulo, Brazil

Dra. Fernanda Scussel - Universidade de Santa Catarina, Brazil

Dr. Glauber Eduardo de Oliveira Santos - Universidade de São Paulo, Brazil

Dr. João Ricardo Freire - Universidade NOVA de Lisboa \& Universidade Europeia, Portugal

Dr. Julio Araújo Carneiro da Cunha - Universidade Nove de Julho, SP, Brazil

Dra. Marina Lourenção - Universidade de São Paulo, Brazil

Ph.D. Candidate: Mateus Artêncio - Universidade de São Paulo, Brazil

Dra. Mariana Sutter - Toulouse Business School, France

Dr. Marco Ocke - Universidade Federal de Santa Catarina, SC, Brazil

Dr. Mihalis Kavaratzis - University of Leicester, England

Dra. Mirna de Lima Medeiros - Universidade Estadual de Ponta Grossa, PR, Brazil

Dra. Monica Franchi Carniello - Universidade de Taubaté, SP, Brazil 
Dr. Rafael Barreiros Porto - Universidade de Brasília, D.F., Brazil

Dr. Ricardo Limongi - Universidade Federal de Goiás, GO, Brazil

Dr. Robert Bowen - Swansea University, Wales, United Kingdom

Dr. Roberto Flores Falcão - Centro Universitário FECAP, SP, Brazil

Ph.D. Candidate: Sergio Czajkowski - UNICuritiba, PR, Brazil

Dra. Suzane Strehlau - Escola Superior de Propaganda e Marketing, SP, Brazil

Dra. Thaysa Nascimento - Universidade Federal do Rio de Janeiro, RJ, Brazil

Dra. Virginia Castro - Universidade Federal da Integração Latino-Americana, PR, Brazil

Dra. Vivian Iara Strehlau - Escola Superior de Propaganda e Marketing, SP, Brazil

Dr. Wesley Moreira Pinheiro - Pontifícia Universidade Católica de São Paulo, SP, Brazil 


\title{
EDITORIAL EDIÇÃO ESPECIAL: OS CAMINHOS FUTUROS DA GESTÃO DE MARCA DE LUGAR: CENÁRIO BRASILEIRO
}

\author{
Fabiana Mariutti \\ Editora Convidada - Brasil \\ Magdalena Florek \\ Editora Convidada - Polônia
}

\section{Como citar}

American Psychological Association (APA)

Mariutti, F., \& Florek, M. (Eds.). (2022, jan./mar.). Os caminhos futuros da gestão de marca de lugar: cenário brasileiro. Revista Brasileira de Marketing - ReMarK, 21(Num. Esp.), 1-8. https://doi.org/10.5585/remark.v21i1.21486.

"Qual lugar?" Todos os lugares, distantes e próximos, grandes e pequenos, desenvolvidos ou em desenvolvimento, estão procurando as melhores soluções e práticas para atender às necessidades e ambições de seus stakeholders internos e externos. Place branding é uma abordagem que apoia os lugares nesses esforços, estrategicamente e de forma interativa. Como Kavaratzis e Florek (2021) afirmam, "no futuro próximo, a comunidade acadêmica global de gestão de marca de lugar precisará abordar uma série de desafios e questões que permanecem sem solução e sem resposta, com implicações tanto para a teoria quanto para a prática" (p. 28). Ao mesmo tempo, acadêmicos e profissionais precisam considerar as diferenças regionais e locais. As perspectivas, possibilidades e condições brasileiras não são exceção.

Esta primeira edição avança na compreensão deste campo de conhecimento ilimitado, imperativo e interdisciplinar. Agradecemos à equipe editorial do Brazilian Journal of Marketing, a todos os autores e revisores que contribuíram para esta edição especial sobre gestão de marca de lugar (Apêndice I. Nota de Agradecimento).

Contextualmente, de acordo com uma análise da literatura internacional das localizações geográficas encontradas nos artigos sobre place branding, entre 2014 e 2018, a Europa é a área dominante nos estudos, seguida pela Ásia e América do Norte que também têm atraído 
significativa atenção dos estudiosos (Lu et al. 2020). Na América do Sul, especificamente no Brasil, as publicações acadêmicas sobre marca de lugar (place brand), gestão de marketing de marca de lugar (place marketing) e gestão de marca de lugar (place branding) ainda são escassas. Daí a necessidade urgente de compreender adequadamente os fenômenos de gestão de marca de lugar e de difundir o seu conhecimento que muitas vezes é ultrapassado pela prática, causando todos os tipos de problemas e decepções. Sem uma base científica sólida, o place branding se tornará e continuará sendo apenas uma tática baseada em resposta.

Portanto, nesta Edição Especial, gostaríamos de destacar o potencial das realizações científicas dos acadêmicos no Brasil e avançar na discussão entre pesquisadores e profissionais brasileiros. Conforme estudado por Artêncio, Mariutti e Giraldi (2020), um panorama temático de cunho acadêmico-científico averiguou os trabalhos apresentados nos principais congressos brasileiros de Administração: SemeAD, EnANPAD (Divisão de Marketing) e EMA nos últimos vinte anos (1999-2019). Com um total de 5.706 estudos, apenas 18 artigos abordam algumas questões relacionadas à gestão de marca de lugar ou gestão de marketing de marca de lugar, com foco principalmente em nível de país (14), e alguns em cidades (2) e regiões (2). Com base na análise dos títulos desses estudos, dos resumos e das palavras-chave identificadas nos anais dos congressos, apenas três trabalhos foram identificados como alinhado à uma designação conceitual de marketing de lugares, apresentados no SemeAD 2016 e no EMA 2018; e um trabalho de gestão de marca de lugar apresentado no EMA 2018. Notavelmente, os estudos são específicos para alguns aspectos da place brand (local de origem, identidade, imagem, reputação e cultura, para citar alguns).

Diante desse retrato científico, conclusões apontadas podem ser complementadas.

Em primeiro lugar, nas pesquisas realizadas no Brasil em nível nacional, os estudos de place branding certamente estão evoluindo. E precisam de tal inserção nacional, uma vez que a marca de lugar pode ser usada como um instrumento no planejamento espacial estratégico para apoiar uma mudança estrutural nos lugares por contribuir para a melhoria da estrutura econômica e social dos lugares e remodelar as respostas aos desafios contemporâneos enfrentados pelos lugares. Referente ao posicionamento de marca ou gestão da marca de um lugar, um espaço ou território não é um conceito absoluto, está sempre relativo a uma esfera de aplicação social-humana ou estrutura econômica-política, o qual se desenvolve potencialmente por meio de processos - possivelmente contestados - para produzir, manter e atribuir espaços com significado, reforça Warnaby (2018). 
Em segundo lugar, há a necessidade de desenvolvimento teórico e empírico dos conceitos de marca de lugar, gestão de marketing de marca de lugar, e gestão de marca de lugar, não apenas com base em estudos sobre cidades, estados e regiões brasileiras, mas também em outros lugares do país como aeroportos, aglomerados industriais, avenidas, parques, praias, praças, museus, teatros etc. Como a diversidade de locus é reconhecida em um país extenso como o Brasil, é fundamental fornecer perguntas de pesquisa sobre a espacialidade existente na literatura de marketing, como enfatizam Giovanardi e Lucarelli (2018), Oliveira (2015) e Warnaby (2018).

Em terceiro lugar, o avanço ontológico e epistemológico das linhas de pesquisa em place branding é necessário para provocar reflexões e direcionamentos para o avanço teórico, particularmente nos grupos de pesquisa brasileiros. Como Govers (2021) afirma, "as agências de financiamento e governos que valorizam que a gestão de marca de lugar seja de importância estratégica, de longo prazo e crucial são, infelizmente, uma pequena minoria” (p.338). Com isso em mente, esperamos chamar a atenção de universidades, pesquisadores e gestores de políticas públicas brasileiras para a importância e relevância da gestão de marca de lugares. Afinal, “A marca de lugar tem sido usada para promover a reestruturação econômica, a inclusão e coesão social, o engajamento e a participação política, a identificação do lugar e o bem-estar geral dos cidadãos", expõe Oliveira $(2015$, p.18).

Os stakeholders per se, que são cocriadores no processo de gestão de marca de lugar (Kalandides, Kavaratzis, \& Boisen, 2012; Mariutti \& Giraldi, 2021) precisam estar envolvidos na gestão de locais públicos e/ou privados, estrategicamente alinhados com as atividades de gestão de marca de lugar para aumentar o valor de um lugar (Mariutti \& Giraldi, 2021). Traçar o escopo da pesquisa ao longo desse caminho estabelece conexões entre prática e teoria e oferece insights sobre os vindouros rumos para lugares peculiares, escondidos ou esquecidos no Brasil.

Para abordar os contextos acima em conjunto com o cenário acadêmico brasileiro, compartilhamos que 27 trabalhos foram submetidos durante os seis meses de pandemia (fevereiro a agosto de 2021); eles foram cuidadosamente revisados e analisados. Além disso, os melhores estudos selecionados pelos líderes de tema de 'Gestão de Marca de Lugar' no Encontro de Marketing - EMA 2021 (por Fabiana Mariutti e Mihalis Kavaratzis) e no EnANPAD 2021 (por Fabiana Mariutti e João Freire) foram convidados para submissão no processo de avaliação regular pela Revista Brasileira de Marketing. Ambas os congressos são organizados pela ANPAD - Associação Nacional de Pós-Graduação e Pesquisa em 
Administração). Pela primeira vez no Brasil, uma trilha acadêmica de place branding aconteceu em congressos brasileiros. Portanto, com base nas avaliações dos revisores e na compreensão do domínio da linha de pesquisa, as decisões foram tomadas pelos editores convidados e editores-chefes da revista. Por fim, foram escolhidos nove manuscritos para publicação.

Contribuições robustas emergiram desta coletânea pioneira. Os seguintes artigos sistematicamente escolhidos ilustram algumas arcabouços e insights para a pesquisa sobre gestão de marca de lugar ser concebivelmente aprimorada na literatura nacional.

A edição começa com um artigo de Carniello e dos Santos que analisam o branding como estratégia de desenvolvimento territorial, discutindo a convergência entre o city branding e um plano diretor municipal no caso da cidade de São Paulo. Foi identificada uma convergência entre os pilares da marca e os conteúdos do plano diretor, especialmente a comunicação como elemento-chave para as proposições sociais e de gestão. Postula-se, assim, uma perspectiva estratégica de place branding com uma abordagem qualitativa, com design documental.

O artigo subsequente, de Kamlot e Santos Vieira de Jesus, discute o cerne do processo de branding, principalmente, a identidade da marca de cidade. O artigo contribui para a exploração das dimensões físicas e simbólicas da identidade da marca do Rio de Janeiro e para a interpretação detalhada do RJ, considerando a evolução recente da cidade. Adota o método qualitativo para análise documental, o conjunto de dados foi combinado por artigos de pesquisa, e-mails, boletins, relatórios de empresas e governos, artigos de jornais etc. Este estudo fornece indicações importantes sobre como delinear políticas públicas a partir da compreensão da identidade de uma cidade.

O próximo artigo analisa os sentimentos que acompanham o conteúdo gerado pelos usuários no Instagram, expressos em hashtags vinculadas à marca da cidade de Pernambuco (capital de Recife). Resultados dessa pesquisa mista (qualitativa e quantitativa) de Lima, Pessanha, Araújo, Alves e Cesário sugerem que estudos utilizando análise de sentimento de conteúdo de usuários em redes sociais podem ser utilizados por gestores públicos para entender o comportamento dos moradores, turistas e potenciais visitantes por meio da pesquisa-ação, preparando e adaptando melhor a cidade.

Beck e Ferasso, por sua vez, analisam o reflexo da identidade da marca, ou seja, a imagem das cidades. Neste estudo, uma revisão sistemática da literatura foi utilizada para analisar o conteúdo da literatura sobre imagem urbana no contexto do Mercado Comum do Sul (MERCOSUL). O trabalho demonstra que a gestão da imagem urbana mostra como as cidades 
podem superar as disparidades socioeconômicas, com a implementação de um projeto eficiente de imagem urbana a fim de promover desenvolvimento urbano bem-sucedido.

Monte, Pompeu e Holanda descrevem uma interessante abordagem qualitativa temáticaexploratória de um lugar esquecido - museu corporativo - como um espaço empresarial e cultural que influencia a imagem de um lugar. Os autores acreditam que os museus corporativos são um importante ativo cultural na promoção da marca do lugar e ainda, que esses museus pertencem não apenas à empresa, mas também à cidade, região e país onde está localizado, atraindo turistas e investidores, fomentando um sentimento de pertencimento entre os moradores. O estudo, que foi apresentado no Place Branding Track no EMA 2021, propõe um modelo explicativo e teórico de como uma marca de lugar (cidade, região ou país) pode ser potencializada por meio de um museu corporativo.

Outro ponto de vista empresarial foi adotado por Munaier, Rocha e Portes ao investigarem o impacto da localização da sede comercial de um prestador de serviços em educação física perante a confiança da marca dos clientes. A teoria da troca social e a confiança na marca foram fundamentos teóricos para as hipóteses deste estudo quantitativo usando técnicas estatísticas multivariadas. Ao perceber que a localização da sede influencia a confiança da marca dos clientes, os autores sugerem que a escolha de um local é um processo complexo que as empresas devem gerenciar com cuidado; ao mesmo tempo, os lugares podem usar essas relações para fins de branding.

As questões de mensuração do processo de gestão de marca de lugar são discutidas por Cunha, Luce e Rovedder; eles desenvolveram e testaram a medida de valor da marca local baseada em stakeholders (SBPBE) do Vale dos Vinhedos, na região Sul do Brasil. Com base nos resultados dessa pesquisa quantitativa a partir dos perfis dos entrevistados, os autores demonstram que quanto mais movimentos e atividades das pessoas em um local, mais positiva tende a ser sua avaliação do valor da marca do lugar. Os resultados desta pesquisa, apresentada no Place Branding Track no EnANPAD 2021, sustentam uma proposta de mensuração para avaliar esses múltiplos stakeholders sobre o posicionamento da marca com base em dados comportamentais.

Os dois últimos artigos indicam as recomendações de estudos e as direções para desenvolvimentos futuros no domínio da pesquisa sobre gestão de marca de lugar.

Com base em uma revisão sistemática da literatura, Campos, Galina e Giraldi estabelecem as diretrizes para futuras estudos interdisciplinares sobre soft power, analisando seu alinhamento teórico-prático com indústrias criativas e gestão de marca de lugar. Após a 
adoção da análise de conteúdo com abordagem teóricas, foram identificadas seis áreas relevantes com breve descrição e referências oblíquas nessa investigação pujante: psicossociologia, mobilidade laboral, planejamento urbano, diplomacia artística, gastrodiplomacia e indicações geográficas. Demonstra-se como o soft power e o place branding revelam uma relação explícita entre si: teoricamente, estrategicamente e politicamente.

Ocke e Platt apresentam uma agenda de pesquisa convidativa, fornecendo alguns apontamentos para acadêmicos, profissionais de branding e profissionais de marketing que ocorrem no escopo da gestado de marca - identidade, imagem e reputação de um lugar. Ao adotar material bibliográfico, sob uma visão geral do nation branding com suas orientações econômicas, políticas e cultural-críticas, em que propostas significantes para o Brasil são sugeridas para a gestão eficaz das marcas de lugares, incluindo: (i) nomeação de um órgão formal para coordenar as ações e atores reconhecendo o papel dos stakeholders nas etapas de tomada de decisão; (ii) cooperação entre governadores e líderes para alcançar objetivos comuns sob um programa único de marca guarda-chuva que beneficia diferentes setores da economia; (iii) a conquista de oportunidades de mercado por meio de ativos identitários competitivos, como a orientação da marca green place; e (iv) aplicação de métodos integrados para mensurar e avaliar os resultados dos esforços de place branding.

Com esta Edição Especial, intitulada "Qual lugar? Os Caminhos Futuros do Place Branding”, esperamos incentivar ações e estimular um debate sobre como a gestão de marca de lugar pode efetivamente melhorar os lugares brasileiros e como o conhecimento oferecido pela comunidade acadêmica pode preparar qualquer tipo de lugar para os próximos desafios. "O que vem depois?" Considerando o papel promissor da gestão de marca de lugar no Brasil, nossas recomendações para estudos futuros destacam temas relacionados com o atual status quo atual do país, ao momento pós-pandemia, a reintegração humana aos lugares a, ainda, questões de sustentabilidade. Estudos sobre a gestão de marca de lugar requer estudos interdisciplinares adicionais, integração de disciplinas, grupos de pesquisa de parcerias internacionais, intercâmbios profissionais, iniciativas público-privadas, mapeamento de clusters, análises em geomarketing e métodos mais sofisticados para avaliação crítica acompanhados de problemas de pesquisa instigantes bem estabelecidos.

\section{Referencias}

Artêncio, M., Mariutti, F., \& Giraldi, J. (2020). 20 Anos de Publicações sobre Marca-de-Lugar no Brasil: um panorama analítico-reflexivo da produção acadêmica em congressos de 
Administração. In: XXIII Seminários em Administração - SemeAD 2020 - Universidade de São Paulo.

Giovanardi, M., \& Lucarelli, A. (2018). Sailing through marketing: A critical assessment of spatiality in marketing literature. Journal of Business Research, 82: 149-159.

Govers, R. (2021). Chapter 19 - Place branding practice and scholarship: where we are and whither, we may be going. In: Papadopoulos, N., Cleveland, M. "Image, Marketing, and Branding of Places and Place-based Brands: The State of the Art", Edward Elgar.

Kalandides, A., Kavaratzis, M., \& Boisen, M. (2012). From 'necessary evil' to necessity: stakeholders' involvement in place branding. Journal of Place Management and Development, 5 (1): 7-19.

Kavaratzis, M., Florek, M. (2021). Chapter 2 - The who, what, where, when, and why of place branding, In: Papadopoulos, N., Cleveland, M. "Image, Marketing, and Branding of Places and Place-based Brands: The State of the Art", Edward Elgar.

Lu, H., Ma, W., Yang, Q., \& Zhao, P. (2020). Exploring the impact of factors influencing case selection in the place branding literature from 2014 to 2018, Journal of Urban Affairs, first online.

Mariutti, F. G., \& Giraldi, J. D. M. E. (2021). Branding cities, regions, and countries: the roadmap of place brand equity. RAUSP Management Journal, 56 (2): 202-216.

Oliveira, E. (2015). Place branding as a strategic spatial planning instrument. Place Branding and Public Diplomacy, 11 (1): 18-33.

Warnaby, G. (2018). Taking a territorological perspective on place branding? Cities, 80: 64-66.

\section{Apêndice I}

Nota de Agradecimento

Nós apreciamos o apoio inesgotável do Editor-Chefe Prof. Dr. Julio Carneiro da Cunha.

Somos gratos aos seguintes revisores, que contribuíram de forma organizada para nossa edição especial:

1. Dr. Antônio Azevedo - Universidade do Minho, Portugal

2. Dra. Beatriz Casais - Universidade do Minho, Portugal

3. Dra. Caroline Finocchio - Universidade Federal do Mato Grosso do Sul, Brasil

4. Dra. Cecilia Pasquinelli - Università degli Studi di Napoli Parthenope, Itália

5. Ph.D. Candidate: Donizete Ferreira Beck - Universidade Nove de Julho, SP, Brasil

6. Dr. Eduardo de Paula e Silva Chaves - Universidade de São Paulo \& Instituto Federal de São Paulo, Brasil 
7. Dra. Fernanda Scussel - Universidade de Santa Catarina, Brasil

8. Dr. Glauber Eduardo de Oliveira Santos - Universidade de São Paulo, Brasil

9. Dr. João Ricardo Freire - Universidade NOVA de Lisboa \& Universidade Europeia, Portugal

10. Dr. Julio Araújo Carneiro da Cunha - Universidade Nove de Julho, SP, Brasil

11. Dra. Marina Lourenção - Universidade de São Paulo, Brasil

12. Ph.D. Candidate: Mateus Artêncio - Universidade de São Paulo, Brasil

13. Dra. Mariana Sutter - Toulouse Business School, França

14. Dr. Marco Ocke - Universidade Federal de Santa Catarina, SC, Brasil

15. Dr. Mihalis Kavaratzis - University of Leicester, Inglaterra, Reino Unido

16. Dra. Mirna de Lima Medeiros - Universidade Estadual de Ponta Grossa, PR, Brasil

17. Dra. Monica Franchi Carniello - Universidade de Taubaté, SP, Brasil

18. Dr. Rafael Barreiros Porto - Universidade de Brasília, D.F., Brasil

19. Dr. Ricardo Limongi - Universidade Federal de Goiás, GO, Brasil

20. Dr. Robert Bowen - Swansea University, País de Gales, Reino Unido

21. Dr. Roberto Flores Falcão - Centro Universitário FECAP, SP, Brasil

22. Ph.D. Candidate: Sergio Czajkowski - UNICuritiba, PR, Brasil

23. Dra. Suzane Strehlau - Escola Superior de Propaganda e Marketing, SP, Brasil

24. Dra. Thaysa Nascimento - Universidade Federal do Rio de Janeiro, RJ, Brasil

25. Dra. Virginia Castro - Universidade Federal da Integração Latino-Americana, PR, Brasil

26. Dra. Vivian Iara Strehlau - Escola Superior de Propaganda e Marketing, SP, Brasil

27. Dr. Wesley Moreira Pinheiro - Pontifícia Universidade Católica de São Paulo, SP, Brasil 